\title{
Forças de caráter: evidências de uma escala e prevalência no Nordeste brasileiro
}

\section{Strengths of character: evidence from a scale and prevalence in the Brazilian Northeast}

Fuerzas de carácter: evidencias de una escala y prevalencia en el nordeste brasileño

\author{
Ricardo Neves Couto ${ }^{1}$, ORCID: 0000-0001-9989-4857 \\ Patrícia Nunes da Fonsêca ${ }^{2}$, ORCID: 0000-0002-6322-6336 \\ Paulo Gregório Nascimento da Silva ${ }^{3}$, ORCID: 0000-0002-2878-309X \\ Paloma Cavalcante Bezerra de Medeiros ${ }^{4}$, ORCID: 0000-0002-5868-8333 \\ ${ }^{1234}$ Universidade Federal da Paraíba, Brasil
}

\begin{abstract}
Resumo: Objetiva-se verificar a prevalência das forças de caráter nos nordestinos, especificamente, reunir novas evidências psicométricas da Escala de Forças de Caráter (EFC). Contou-se com uma amostra de 383 pessoas $\left(M_{\text {idade }}=27,81 ; D P=10,83\right)$ oriunda em sua maioria da Paraíba, Brasil $(24,3 \%)$, do sexo feminino $(65,4 \%)$ e solteira $(73,1 \%)$, que responderam a EFC e questões demográficas. Uma Análise Fatorial Exploratória indicou a unidimensionalidade da escala, as 24 forças saturando no fator com valor próprio igual a 9,69, explicando $43 \%$ da variância e cargas fatoriais de 0,41 (perdão) até 0,72 (vitalidade). $\mathrm{O}$ alfa de Cronbach $(0,94)$ e ômega de McDonald $(0,95)$ indicaram precisão satisfatória. Ademais, estatísticas descritivas foram realizadas identificando as forças mais prevalentes no Nordeste, respectivamente: gratidão, bondade, curiosidade, imparcialidade, amor ao aprendizado e esperança. Discute-se que a EFC reúne evidências para sua utilização e se discute a influência da cultura e região na prevalência das forças.
\end{abstract}

Palavras-chave: forças de caráter; virtudes; testes psicológicos; psicologia positiva; psicologia social.

Abstract: This study assesses the prevalence of character strengths among Northeastern Brazilians to, more specifically, gather new psychometric evidence by applying the Character Strengths Scale (CSS) to a sample of 383 people $\left(M_{\text {age }}=27.81 ; S D=10.83\right)$, mostly single (73.1\%) women (65.4\%) from Paraíba, Brazil (24.3\%), who completed the CSS and answered demographic questions. Exploratory factor analysis indicated the unidimensionality of the scale, with the 24 strengths of character loading on the factor with an eigenvalue of 9.69, explaining $43 \%$ of the variance, and with factor loadings ranging from 0.41 (forgiveness) to 0.72 (zest). Cronbach's alpha (0.94) and McDonald's omega (0.95) indicated satisfactory scale reliability. Furthermore, descriptive statistics were calculated to identify the most prevalent strengths in the Brazilian Northeast, namely, gratitude, kindness, curiosity, fairness, love of learning and hope, in decreasing order. Evidence is presented for the use of the CSS as well as the influence of culture and region on the prevalence of character strengths.

Keywords: character strengths; virtues; psychological tests; positive psychology; social psychology. 
Resumen: Se estudia la prevalencia de las fuerzas de carácter en los nordestinos, específicamente con la intención de reunir evidencias psicométricas de la Escala de Fuerzas de Carácter (EFC). Se contó con una muestra de 383 personas $\left(M_{e d a d}=27.81, D E=10.83\right)$ oriunda en su mayoría de Paraíba, Brasil $(24.3 \%)$, del sexo femenino $(65,4 \%)$ y soltera $(73,1 \%)$, que respondieron a EFC y cuestiones demográficas. Un Análisis Factorial Exploratorio indicó la unidimensionalidad de la escala, las 24 fuerzas saturando en el factor con valor propio igual a 9.69, explicando el $43 \%$ de la varianza y cargas factoriales de .41 (perdón) hasta .72 (vitalidad). El alfa de Cronbach= .94 y omega de McDonald= .95 indican precisión satisfactoria. Posteriormente, estadísticas descriptivas fueron realizadas identificando como las fuerzas más prevalentes en el Nordeste: gratitud, bondad, curiosidad, imparcialidad, amor al aprendizaje y esperanza. Se plantea que la EFC reúne evidencias psicométricas para su utilización y se discute la influencia de la cultura y región en la prevalencia de las fuerzas.

Palabras-clave: fuerzas de carácter; virtudes; pruebas psicológicas; psicología positiva; psicología social.

Recebido: 30/03/2020

Aceito: $27 / 10 / 2020$

Como citar:

Couto, R. N., Fonsêca, P. N. da, Silva, P. G. N. da \& Medeiros, P.C.B. (2021). Forças de caráter: evidências de uma escala e prevalência no Nordeste brasileiro. Ciencias Psicológicas, 15(1), e2357. Doi: https://doi.org/10.22235/cp.v15i1.2357

Correspondência: Ricardo Neves Couto. E-mail: r.nevescouto@gmail.com; Rua caiapos, n. 107, Conjunto Esperança 1; Parnaíba-PI, Brasil CEP: 64215-470.

O que há de bom nas pessoas tem despertado interesse de pesquisadores e profissionais da área das ciências sociais e saúde. O estudo das virtudes e forças de caráter é exemplo disso, pois tal construto tem ocupado um papel de destaque no campo da Psicologia Positiva (Noronha \& Campos, 2018; Petkari \& Ortiz-Tallo, 2016). As virtudes dizem respeito às capacidades individuais particulares relacionadas aos pensamentos, sentimentos e ações que levam o indivíduo a fazer o que é certo, e que envolvem as forças específicas como rotas para serem atingidas (Peterson \& Seligman, 2004). Já as forças são caracterizadas como pontos fortes preexistentes e autênticos do ser humano, pois proporcionam a sobrevivência da espécie de maneira positiva (Noronha \& Zanon, 2018; Seligman \& Csikszentmihalyi, 2000).

Ademais, o uso das forças de caráter é socialmente valorizado, estando relacionado positivamente com traços positivos da personalidade (e.g., amabilidade e extroversão) (Couto \& Fonsêca, 2019; Noronha \& Campos, 2018). Além de ser uma importante condição para uma usufruir de educação, controle de excessos, experienciar autorregulação emocional e satisfação com a vida (Harzer \& Ruch, 2013; Noronha \& Batista, 2020a), além de ser essencial para um clima interpessoal e grupal agradável, com maiores percepções de bem-estar e saúde mental (Haridas, Bhullar, \& Dunstan, 2017).

Em levantamento feito por Reppold, Gurgel e Schiavon (2015), até o ano de 2014, nenhuma referência foi encontrada no Brasil acerca de forças de caráter. Já Pires, Nunes e Nunes 
(2015), até o mesmo ano de 2014 não acharam nenhum instrumento publicado baseado no modelo de forças e virtudes.

Contudo, recentemente foram publicados alguns estudos que versam sobre o estudo das forças de caráter no Brasil (Couto \& Fonseca, 2019; Noronha \& Batista, 2017; 2020a; 2020b; Noronha \& Martins, 2016; Noronha, Silva, \& Rueda, 2018; Seibel, DeSousa, \& Koller, 2015). Com destaque para o de Noronha, Dellazzana-Zanon e Zanon (2015), no qual apresentam excelentes evidências psicométricas da Escala de Forças de Caráter (EFC), construída de maneira parcimoniosa para o contexto brasileiro. Logo, nota-se que o estudo desse construto ainda é incipiente. Porém, cada vez mais ganha notoriedade nacional, facilitado por instrumentos de avaliação que permitem conhecer o ranking dos pontos fortes de cada região.

Caracterizado por sua natureza autêntica e potencialidade de presença em todas as pessoas, o ranking é dinâmico e influenciado pelas particularidades de cada região (Peterson \& Seligman, 2004; Seibel et al., 2015). Nessa linha, evidencia-se que conhecer as prevalências das forças de cada país é um suporte básico para resolução de problemas sociais cotidianos (Romero, Guajardo, \& Sánchez, 2016) e exaltação da base da psicologia positiva (emoções, qualidades e instituições positivas) (Noronha \& Batista, 2017).

As forças de caráter podem se manifestar através de comportamentos, pensamentos e/ou sentimentos, são autenticas e permitem o funcionamento mais ideal possível para o ser humano (Noronha et al., 2018; Peterson \& Seligman, 2004). Assim, à medida que as pessoas fazem uso das forças, tornam-se virtuosas (Oliveira, Nunes, Legal, \& Noronha, 2018).

Após investigação na literatura, incluindo as tradições filosóficas e religiosas, foi elaborada uma classificação denominada Values in Action (VIA), a qual reúne as 24 forças de caráter englobadas teoricamente em seis virtudes a fim de descrever as potencialidades dos indivíduos. Para tanto, os autores definiram 10 critérios para que uma característica psicológica seja incluída como força de caráter (Park, Peterson, \& Seligman, 2006; Peterson \& Seligman, 2004; Seligman, Steen, Park, \& Peterson, 2005). Resumidamente, Freitas, Barbosa e Neufeld (2016) transcrevem os critérios reunidos pelos autores. Todos, ou pelo menos a maior parte deles deve ser satisfeita: (1) ser útil e colaborar para uma vida boa para si e os demais, (2) proporcionar mais do que o resultado próprio para si, sendo o seu uso moralmente valorizado, (3) sua execução não rebaixa as outras pessoas, (4) tem antônimos óbvios que são opostos e negativos, (5) expressões estáveis e capazes de ser mensuradas, (6) cada força é distinta de outras, (7) englobadas em modelos consensuais, (8) presentes em todas as faixas etárias, (9) capacidade de seleção e total nulidade em algumas pessoas e (10) formadas em instituições, é alvo de práticas sociais e rituais que cultivam e mantêm os pontos fortes.

Acrescenta-se que ao usar as 24 forças, o indivíduo pode experienciar a sensação de excitação, anseio, descobertas e revigoração, além de se tornar uma pessoa reconhecidamente com um bom caráter (Seijts, Crossan, \& Carleton, 2017). Para tanto, de maneira geral, espera-se que socialmente os pontos fortes sejam colocados em prática, almejando as seis virtudes teoricamente propostas e, consequentemente, obter boas relações sociais, capacidade de lidar com situações difíceis, aquisições de novas aprendizagens e funcionamento saudável (Noronha \& Campos, 2018)

Em síntese, o modelo apresentado na Tabela 1 foi proposto para examinar e descrever minuciosamente o que há de melhor em seres humanos, facilitar a identificação, a valorização e a prática cotidiana das forças, apresentando como condição a possibilidade de mensuração. Evidencia-se que essa organização serve como orientação ao leitor e pesquisador, no entanto, a classificação deve ser encarada como descritiva, e não prescritiva (Niemiec, 2013). 
Tabela 1- Descrição das Forças e Agrupamento em Virtudes, Traduzida para Português Brasileiro (adaptado de Noronha \& Barbosa, 2016, pp. 23-25)

\begin{tabular}{|c|c|}
\hline Virtudes & Descrição das forças \\
\hline & $\begin{array}{l}\text { Criatividade: ficar aberto para pensamentos e diferentes formas de fazer as } \\
\text { coisas de maneira produtiva, com originalidade e inventividade. } \\
\text { Curiosidade: Busca por novidades, experiências e interesse, incluindo } \\
\text { aprofundamento em um conhecimento ou assunto genérico e diverso. } \\
\text { Pensamento crítico: procurar por evidências que vão contra crenças e } \\
\text { planos, pesando de forma justa, proporciona a oportunidade de mudar de } \\
\text { ideia. } \\
\text { Amor ao aprendizado: maneira que a pessoa assimila informações e } \\
\text { habilidades novas de forma genérica. } \\
\text { Sensatez: ter discernimento nas situações, oferecer sábios conselhos, com } \\
\text { um olhar que faça sentido para si e os outros. }\end{array}$ \\
\hline Coragem & $\begin{array}{l}\text { Bravura: Enfrentar as adversidades sem medo, com convicção, baseando- } \\
\text { se no que acredita ser certo, não temer ameaças, desafios e até dor. } \\
\text { Perseverança: contínua ação voluntário em direção a um objetivo atingível, } \\
\text { mesmo com obstáculos, dificuldades ou desencorajamento. } \\
\text { Autenticidade: expressar-se genuinamente, sentimentos e ações } \\
\text { verdadeiras. } \\
\text { Vitalidade: Entusiasmo de viver, sentir-se ativo, animado, com energia e } \\
\text { vigor. Indicativo de saúde ligado a fatores psicológicos e somáticos. }\end{array}$ \\
\hline Humanidade & $\begin{array}{l}\text { Amor: Troca de ajuda, consolo e aceitação, envolve sentimentos positivos } \\
\text { fortes, compromisso e até mesmo sacrifício. } \\
\text { Bondade: Estar atento ao próximo, fazer boas ações, inclui generosidade, } \\
\text { cuidado, compaixão, amor altruísta e delicadeza. } \\
\text { Inteligência Social: Processar informação emocional e agir sabiamente nos } \\
\text { relacionamentos, identificando o conteúdo para facilitar as interações. }\end{array}$ \\
\hline Justiça & $\begin{array}{l}\text { Cidadania: responsabilidade social, lealdade e trabalho em equipe, com o } \\
\text { objetivo do bem comum, para além do interesse pessoal. } \\
\text { Imparcialidade: tratar as pessoas de modo similar ou idêntico, sem deixar } \\
\text { que sentimentos ou questões pessoais influenciem as decisões. } \\
\text { Liderança: integração de atributos que orientam a influência para ajudar os } \\
\text { outros, dirigir e motivas ações para o sucesso coletivo. }\end{array}$ \\
\hline Temperança & $\begin{array}{l}\text { Perdão: mudanças pró-sociais dentro do indivíduo que foi ofendido ou } \\
\text { prejudicado por um parceiro de relacionamento. } \\
\text { Modéstia: deixar que ações falem por si, não buscar destaque. Acreditar } \\
\text { que não é melhor que as outras pessoas. } \\
\text { Prudência: ter cuidado com suas escolhas, não assumir riscos indevidos, } \\
\text { não dizer ou fazer coisas que causem arrependimento futuro. } \\
\text { Autoregulação: exercer controle sobre suas respostas (impulsos e } \\
\text { emoções), a fim de atingir objetivos e viver nos padrões. }\end{array}$ \\
\hline Transcendência & $\begin{array}{l}\text { Apreciação do belo: perceber a beleza, excelência e/ou desempenho de } \\
\text { habilidades nos domínios do cotidiano: natureza, arte, matemática e ciência. } \\
\text { Gratidão: sensação de agradecimento e alegria em resposta ao recebimento. } \\
\text { Esperança: aguardar, acreditar e trabalhar para um futuro melhor. } \\
\text { Humor: visão alegre e leve da adversidade, sustentando o bom humor. } \\
\text { Espiritualidade: crenças coerentes a respeito do significado do universo e } \\
\text { do seu lugar nele, além de uma crença em um propósito maior. }\end{array}$ \\
\hline
\end{tabular}


A partir do modelo teórico proposto, foi construído o 240-item VIA Inventory of Strengths (VIA-IS), com 10 itens para cada força, totalizando 240 itens, como primeiro passo para investigação empírica das forças (Peterson \& Seligman, 2004). Mundialmente utilizado, constata-se que o modelo hexafatorial, todavia, não foi encontrado em estudos empíricos que exploraram a dimensionalidade do construto; no estudo original, a estrutura se apresentou com cinco fatores (Peterson \& Seligman, 2004), semelhante aos estudos de Azañedo, FernándezAbascal e Barraca (2014) e Ruch, Martínez-Martí, Proyer e Harzer (2014). Contudo, estruturas com quatro (Brdar \& Kashdan, 2010) e três (McGrath, 2015) dimensões já foram encontradas.

No Brasil, esse instrumento foi adaptado e validado por Seibel et al. (2015), comportandose com uma estrutura unifatorial. Noronha et al. (2015) consideraram o modelo VIA para elaborarem a Escala de Forças de Caráter (EFC), um instrumento brasileiro mais parcimonioso que avalia as forças e reúne evidências de validade interna (Noronha \& Batista, 2020a) e com variáveis externas, tais como estilos parentais (Noronha \& Batista, 2017), personalidade (Noronha \& Campos, 2018) e autorregulação emocional (Noronha \& Batista, 2020b). A princípio foram construídos três itens para cada força, porém a força apreciação do belo, após passar pela análise dos juízes, ficou composta por apenas dois, totalizando uma escala com 71 itens. Os autores, por meio de análises robustas de retenção de fatores (e.g. método Hull, Análise paralela), identificaram a unidimensionalidade da EFC, corroborando com o modelo achado por Seibel et al. (2015) usando o VIA-IS.

Com isso é evidenciada a variabilidade do modelo quanto à cultura, porém, a possibilidade de se estudar as forças de maneira específica (Noronha \& Martins, 2016) e, também, de ser feita uma avaliação global (Neto, Neto \& Furnham, 2014). Contudo, Noronha e Zanon (2018) reúnem diferentes evidências psicométricas da EFC, encontrando por meio de uma análise fatorial exploratória uma estrutura de três fatores, em uma amostra de 981 universitários do sudeste brasileiro.

Dessa forma, observa-se que internacionalmente o estudo de forças e virtudes tem destaque, porém a estrutura teórica de seis fatores proposta originalmente por Peterson e Seligman (2004) não tem sido replicada empiricamente. Fato este que não impede avanços na área e a refinação das escalas propostas para a avaliação dos pontos fortes do indivíduo. Especificamente, a EFC tem demonstrado evidências psicométricas satisfatórias, conforme supracitado e merece o esforço a fim de buscar reunir novos achados em diferentes regiões do Brasil.

Contudo, deve-se ressaltar que independente do instrumento utilizado, o estudo das forças de caráter é executado de forma independente, podendo a avaliação ser feita de maneira geral, devido a influência mútua e a inter-relação entre as forças (Noronha \& Barbosa, 2016; Seibel et al., 2015). Ademais, parte-se do pressuposto de que as 24 forças são universais e todos os indivíduos apresentam a capacidade de desenvolver e colocar em prática, a fim de superar adversidades e fatores de risco, que afetam negativamente o bem-estar e saúde mental (Haridas et al., 2017).

Park et al. (2006), e McGrath (2015) realizam pesquisas com amostra oriundas de diferentes países, incluindo o Brasil, e acrescentam evidências da universalidade do modelo. Porém, ressaltam a impossibilidade de fazer generalizações e a característica individual das forças, a qual é influenciada por particularidades de cada região. Sobretudo, por conta das diferenças culturais, como investigou Hofstede (1991) apresentando seu modelo de dimensões culturais que interferem nos comportamentos de cada indivíduo a partir de cada perspectivas de valores (distância ao poder, individualismo versus coletivismo, masculinidade versus feminilidade, aversão à incerteza e orientação a longo prazo versus orientação a curto prazo). 
Especificamente, no que diz respeito ao modelo de dimensões culturais de Hofstede (1991), Hofstede, Hofstede \& Minkov (2010) identificaram que o Brasil pontua alto em distância ao poder, sugerindo que os indivíduos aceitam bem hierarquias. Pontua mais em características coletivistas, definindo as pessoas como interligadas; apresentam características da masculinidade como a competição em busca de seus objetivos, porém prevalece a qualidade de vida. Chama-se atenção também para o alto nível de aversão a incerteza, criando regras e sistemas jurídicos. Por fim, na dimensão orientação a longo prazo versus curto prazo, o Brasil demonstrou que sua população aceita mudanças facilmente por acreditar que fazem parte da vida (Santana, Mendes, \& Mariano, 2014).

Nessa linha, são encontrados estudos internacionais que foram realizados com o objetivo de identificar a prevalência das forças de caráter em diferentes lugares. Por exemplo, na Espanha (Azañedo et al., 2014) e Noruega (Boe, Bang, \& Nilsen, 2015). Ademais, destaca-se o estudo realizado no México (Romero et al., 2016), devido a sua cultura latino-americana semelhante à do Brasil. Na ocasião os autores discutem que a prevalência encontrada (gratidão e bondade) reflete a relação que o povo mexicano tem com as pessoas, priorizando as interpelações sociais de maneira coletivista.

No Brasil, estudos similares foram realizados por Seibel et al. (2015) utilizando para mensuração das forças o VIA-IS e Noronha et al. (2015) por meio da EFC. Contudo, ressalta-se que neste último estudo, a amostra foi composta unicamente por estudantes universitários oriundos de São Paulo e Minas Gerais. Sugere-se, então, que diferentes estudos sejam realizados nas demais regiões do Brasil, com amostras mais heterogéneas a fim de colaborar com a identificação do perfil de pontos fortes do brasileiro, além de proporcionar maiores indicadores acerca do modelo originalmente proposto, testando a universalidade e a influência cultural no ranking.

Tendo em vista o supracitado, a presente investigação tem a intenção de ampliar o arcabouço teórico e empírico acerca dos pontos fortes da população brasileira. Considerando as características continentais do país e sua relação na influência do construto abordado, questionase: Quais os índices psicométricos da EFC no Nordeste brasileiro? Qual a prevalência das forças de caráter dos residentes da região Nordeste? A fim responder essas perguntas, objetiva-se de maneira geral verificar a prevalência das forças de caráter nos nordestinos, especificamente, reunir novas evidências psicométricas da EFC. Para tanto, é realizado um estudo empírico com pessoas oriundas dos nove estados do Nordeste, tenha-se em conta que a escala, de maneira parcimoniosa, já demonstra índices psicométricos aceitáveis e a característica da amostra é inédita. Logo, pretende-se reunir novas evidências de validade e precisão da medida, ampliando sua potencial aplicabilidade, além de verificar os pontos fortes que configuram o caráter do nordestino.

\section{Materiais e Método}

\section{Participantes}

Tendo critério de inclusão o participante ter idade mínima de 18 anos completos e ser residente em uma cidade da região Nordeste, contou-se com 383 respondentes com idade variando entre 18 e 72 anos $(M=27,81 ; D P=10,03)$ oriundos, em sua maioria, dos Estados da Paraíba (34,3\%), Piauí $(22,7 \%)$ e Pernambuco (13,5\%), do sexo feminino $(65,4 \%)$, solteiros $(73,1 \%)$, com nível superior incompleto $(46 \%)$ e sem filhos $(74,3 \%)$.

\section{Instrumentos}

Escala de Forças de Caráter (EFC): Proposta e validada originalmente no contexto da região sudeste do Brasil (Noronha et al., 2015), é uma escala com 71 itens (e.g., item 39. Sei 
admirar a beleza que existe no mundo; item 40. Não desisto antes de atingir as minhas metas) que avaliam as 24 forças de caráter. Essas são representadas pela somatória de três itens específicos para cada ponto forte, exceto apreciação do belo, que na construção ficou apenas com dois itens. Respondidos em uma escala tipo Likert, variando desde 0 "nada a ver comigo" a 4 "tudo a ver comigo", uma maior pontuação sugere a presença do ponto forte avaliado no cotidiano do indivíduo. No estudo de elaboração, os autores encontraram índices psicométricos satisfatórios (estrutura unifatorial, com alfa de Cronbach igual a 0,93).

Questionário sociodemográfico. Conjunto de questões (e.g., idade, sexo, estado em que reside, estado civil, renda, filhos) a fim de caracterizar os participantes da pesquisa.

\section{Procedimento}

O estudo foi executado após a aprovação do projeto pelo Comitê de Ética em Pesquisa de uma instituição pública do nordeste brasileiro (CAA: 77974517.8.0000.5188; $\mathrm{n}^{\circ}$ protocolo: 2.350.522), em que foram respeitadas as resoluções do Conselho Nacional de Saúde 466/12 e 510/16. Em ambas as formas de coleta, as pessoas eram convidadas a participar da pesquisa de forma voluntária e anônima, e informadas dos objetivos, das características da investigação e da possibilidade de desistência, sem ônus. Ademais, era comunicado os riscos que os participantes poderiam sofrer durante a aplicação, como, por exemplo, constrangimento, durante a leitura de alguns itens. Após as informações, a aplicação que, em média, durava 10 minutos, foi admitida ao concordarem e assinarem o Termo de Consentimento Livre e Esclarecido.

Operacionalmente, os livretos contendo os instrumentos foram aplicados presencialmente, por colaboradores devidamente treinamentos que na ocasião visitaram residências, selecionadas aleatoriamente em algumas cidades do Nordeste, além de contar com a participação de pessoas presentes em locais públicos de grande circulação, tais como shoppings e praças. Ademais, a coleta também ocorreu de forma online, sendo os instrumentos disponibilizados em redes sociais e e-mails dos participantes. Ressalta-se que as aplicações (presencial e virtual) apresentam resultados similares em pesquisas científicas (Brock, Barry, Lawrence, Dey, \& Rolffs, 2012).

\section{Análise de dados}

Com o Amos, versão 23, foi executada uma análise fatorial confirmatória, na qual, os seguintes indicadores foram utilizados para avaliar a adequação do modelo (Tabachnick \& Fidell, 2013): (1) Comparative Fit Index (CFI); (2) Tucker-Lewis Index (TLI) (3) Root-Mean-Square Error of Approximation (RMSEA) e seu intervalo de confiança de 90\% (IC90\%). Com o SPSS, versão 23, foram executadas estatísticas descritivas e de dispersão a fim de caracterizar a amostra e descrever a prevalência das forças de caráter, além do alfa de Cronbach, a fim de obter a precisão da EFC. Com o Factor 10.3 (Lorenzo-Seva \& Ferrando, 2013) foram realizados os testes KMO e o Bartlett para verificar a possibilidade de fatoração da matriz de dados, seguidos de uma análise fatorial ULS, método Hull, para conhecer a estrutura fatorial da escala no contexto do Nordeste brasileiro, além do ômega de McDonald para maior robustez de fidedignidade.

\section{Resultados}

Inicialmente, as 24 forças de caráter foram computadas a partir de parcelas de itens como sugere a literatura (Noronha \& Barbosa, 2016; Noronha \& Batista, 2020b). Assim, para cada indicador foi necessário somar os itens que representavam cada força (e.g., os três itens que mensuravam bondade foram somados); nessa linha, testou-se o modelo hexafatorial baseado nas seis virtudes teoricamente propostas a partir de uma Análise Fatorial Confirmatória, a qual 
sugeriu que a estrutura na amostra aqui considerada não apresenta bons índices de ajustes [CFI= 0,79, TLI $=0,76$, RMSEA $=0,10$ (IC 90\% =0,09-0,12)]. Para considerar a característica de autenticidade e influência de cada região na organização das forças, opta-se por realizar uma análise fatorial exploratória utilizando as 24 forças de caráter para investigar o modelo fatorial da EFC no Nordeste brasileiro.

Quanto à definição da quantidade de fatores, foi resguardada pela adequação dos índices de fatorabilidade da matriz de dados $[\mathrm{KMO}=0,93$; teste de Bartlett $(p<0,05)]$. Uma análise fatorial ULS, utilizando um dos mais adequados e eficientes métodos de retenção de fatores, o Hull, além da Análise Paralela (Damásio, 2012; Lorenzo-Seva, Timmerman, \& Kiers, 2011), os quais indicaram a unidimensionalidade da escala, Os resultados acerca das evidências psicométricas da EFC são descritos na Tabela 1, acrescidos das prevalências das forças de caráter.

Tabela 2

Estatísticas descritivas e estrutura fatorial da Escala de Forças de Caráter

\begin{tabular}{|c|c|c|c|c|}
\hline Variáveis & $M$ & $D P$ & $\begin{array}{c}\text { Carga } \\
\text { Fatorial }\end{array}$ & $h^{2}$ \\
\hline 01. Criatividade & 7,46 & 2,23 & $0,65 *$ & 0,43 \\
\hline 02. Curiosidade & 9,50 & 2,15 & $0,62 *$ & 0,38 \\
\hline 03. Pensamento Crítico & 8,86 & 2,11 & $0,59 *$ & 0,35 \\
\hline 04. Amor ao aprendizado & 9,11 & 2,34 & $0,68 *$ & 0,46 \\
\hline 05. Sensatez & 7,27 & 2,40 & $0,62 *$ & 0,38 \\
\hline 06. Bravura & 7,75 & 2,59 & $0,68 *$ & 0,46 \\
\hline 07. Perseverança & 8,94 & 2,43 & $0,68 *$ & 0,47 \\
\hline 08. Autenticidade & 8,62 & 2,14 & $0,52 *$ & 0,27 \\
\hline 09. Vitalidade & 7,96 & 2,73 & $0,72 *$ & 0,52 \\
\hline 10. Amor & 8,22 & 2,43 & $0,60 *$ & 0,36 \\
\hline 11. Bondade & 9,60 & 1,99 & $0,70 *$ & 0,49 \\
\hline 12. Inteligência social & 7,94 & 2,31 & $0,66^{*}$ & 0,44 \\
\hline 13. Cidadania & 8,50 & 2,26 & $0,82 *$ & 0,67 \\
\hline 14. Imparcialidade & 9,22 & 1,87 & $0,62 *$ & 0,38 \\
\hline 15. Liderança & 7,43 & 2,52 & $0,70 *$ & 0,49 \\
\hline 16. Perdão & 6,91 & 2,93 & $0,41 *$ & 0,17 \\
\hline 17. Modéstia & 9,10 & 2,05 & $0,60 *$ & 0,37 \\
\hline 18. Prudência & 8,55 & 2,40 & $0,59 *$ & 0,35 \\
\hline 19. Autorregulação & 7,07 & 2,58 & $0,50 *$ & 0,25 \\
\hline 20. Apreciação do belo & 6,36 & 1,49 & $0,62 *$ & 0,38 \\
\hline 21. Gratidão & 9,68 & 2,24 & $0,68 *$ & 0,46 \\
\hline 22. Humor & 7,74 & 2,79 & $0,67 *$ & 0,45 \\
\hline 23. Esperança & 9,11 & 2,49 & $0,65 *$ & 0,42 \\
\hline 24. Espiritualidade & 9,02 & 3,06 & $0,53 *$ & 0,28 \\
\hline Quantidade de itens & & & 24 & \\
\hline Variância explicada (\%) & & & $43 \%$ & \\
\hline Valor próprio & & & 9,69 & \\
\hline$\alpha$ de Cronbach & & & 0,94 & \\
\hline$\Omega$ de McDonald & & & 0,95 & \\
\hline
\end{tabular}

Nota: * carga fatorial considerada satisfatória, isto é, $>|0.30| . \mathrm{h}^{2}=$ comunalidade. $\alpha=$ alfa de Cronbach. $\Omega=$ ômega. 
Como observado na Tabela 2, todas as 24 forças saturaram satisfatoriamente no fator com valor próprio igual a 9,69, explicando $43 \%$ da variância total, com cargas fatoriais acima do recomendado $(>0,30)$, variando de 0,41 (perdão) até 0,72 (vitalidade). Ademais, é possível observar também índices que indicam precisão satisfatória ( $\alpha$ Cronbach $=0,94 ; \Omega$ de McDonald $=$ $0,95)$. Ademais, as forças mais presentes nas vidas dos nordestinos da amostra considerada foram gratidão e bondade, seguidas de curiosidade, imparcialidade, amor ao aprendizado e esperança. Ademais, destaca-se que a força espiritualidade teve o maior desvio padrão, indicando uma maior dispersão e variabilidade das pontuações.

\section{Discussão}

A presente pesquisa buscou investigar a prevalência dos pontos fortes dos nordestinos, testando o modelo em seis virtudes e reunindo novas evidências psicométricas da escala EFC. Observa-se, pois, que os objetivos propostos foram alcançados e a EFC apresentou uma estrutura com um fator, com índices de precisão igualmente satisfatórios para pesquisas no contexto da região Nordeste (Cohen, Swerdlik, \& Sturman, 2014; Nunnally, 1978).

A estrutura fatorial de seis fatores, pensada teoricamente para organizar as forças, não foi confirmada na amostra do presente estudo. Dessa forma, optou-se por explorar os dados. A estrutura unidimensional, encontrada a partir de uma análise exploratória, corrobora com a originalmente achada pelos autores (Noronha et al., 2015) e com o modelo de forças de caráter demonstrado por Seibel et al. (2015) no contexto brasileiro. Esse fato já aconteceu em diferentes países, onde já foram encontrados modelos alternativos ao original (Brdar \& Kashdan, 2010; McGrath, 2015; Noronha \& Zanon, 2018). O certo é que as diferenças de modelos representam a natureza mutável dos pontos fortes dos indivíduos e sua passividade à cultura, tornando assim a reunião em diferentes modelos uma tarefa teórica. A tarefa empírica de avaliar as 24 forças pautadas em um modelo unidimensional, propõe que essa tarefa pode ser realizada de maneira global e/ou cada uma de maneira específica (Neto et al., 2014; Noronha \& Martins, 2016).

Logo, destaca-se que as forças são inter-relacionadas e mesmo que possam ser encontradas universalmente em diferentes culturas, o esperado é que seu ranking seja particular do contexto, sendo possível avaliar cada uma de maneira individual (McGrath, 2015, Noronha et al., 2018). Assim, avança-se nos objetivos do presente estudo e é identificada a prevalência das forças de caráter da região Nordeste brasileira, com maiores magnitudes, respectivamente: gratidão, bondade, curiosidade, imparcialidade, amor ao aprendizado e esperança. Esses resultados, em parte, coincidiram com os do Sudeste (Gratidão, curiosidade, esperança, bondade e justiça) de Noronha et al., 2015. Logo, os participantes desses estudos demonstraram ser gratos por tudo que a vida proporciona, ter cuidado e preocupação com os outros, buscar novas possibilidades e maneiras para lidar com as situações, além de uma conexão, exploração do universo e pensamento esperançoso, mesmo com dificuldades diárias, reunindo assim aspectos fortes do caráter. Além disso, um maior desvio padrão encontrado na força espiritualidade demonstrou uma maior variabilidade dos dados, sugerindo que há nordestinos que experienciam muito a espiritualidade, enquanto há outros que ainda não fazem uso dessa força.

Aproximando-se dos resultados encontrados no México (Romero et al., 2016) fica clara a representação da cultura latino-americana, a qual é caracterizada por ser mais coletiva do que individualista (Díaz-Loving, 2005). Evidenciam-se as características positivas quanto ao tratamento similar de todas as pessoas e a não preocupação com o destaque individual. Sendo possível identificar similaridades com um perfil proposto por Hofstede et al. (2010) para o Brasil, 
o qual se caracteriza pela preocupação com o bem-estar comum acima do pessoal. Assim, as forças de caráter prevalentes nas amostras brasileiras convergem com as características apresentadas um modelo proposto para a psicologia social. Uma vez que, segundo Hofstede (1991) e Hofstede et al. (2010) no Brasil, as pessoas aceitam bem hierarquias, buscam competição, mas priorizam a qualidade de vida, além de criarem regras como aversão a incerteza e aceitar mudanças facilmente por acreditar ser parte da vida (Santana et al., 2014).

Ressalta-se a importância da temática e a discussão de maneira especifica dos pontos fortes encontrados no Nordeste brasileiro, dado que o fator histórico da região, marcado por superações, adversidades geográficas, fases de secas, localidades que a água é escassa, necessidades básicas e baixo investimento econômico (Gonçalves \& Araújo, 2015), pode ser apontado como um fator que contribuiu para a prevalência das forças identificadas. Para a superação, faz-se necessário a união dos membros da região, reconhecimento e valorização do que se tem e, sobretudo, devido a essas particularidades da região Nordeste, a população continuar sempre com a esperança de dias melhores, pois, as experiencias de superação já passadas sugerem boas perspectivas. Isso faz com que os indivíduos se engajem em suas atividades diárias, mantendo sua qualidade de vida com o que é oferecido e alcançado.

Com esses resultados, acredita-se que informações acerca do perfil positivo do brasileiro foram acrescentadas, de maneira mais holística, reuniram uma amostra mais heterógena (diferentes faixas etárias e escolaridade), diferenciando-se dos estudos realizados no contexto brasileiro, os quais contaram com amostras unicamente da região Sudeste e estudantes de graduação (Noronha \& Batista, 2017; 2020a; 2020b; Noronha et al., 2015; Noronha \& Zanon, 2018;). Destaca-se assim a sua importância, na medida que estudos transculturais, que contaram com o Brasil, agruparam realidades por semelhanças culturais, porém ressaltaram a influência e passividade das características de cada região (McGrath, 2015; Park et al., 2006). Nessa conjectura, deve-se levar em conta a dimensionalidade continental e variabilidade cultural do Brasil.

Nessa linha, reconhece-se a limitação da amostra aqui utilizada, especifica de uma das cinco regiões brasileiras e de natureza não probabilística, através de uma coleta por conveniência, não sendo possível uma generalização dos resultados. Fato que se deve, também, pelo delineamento não experimental da pesquisa (Breakwell, Hammond, Fife-Chaw, \& Smith, 2010). Porém, evidencia-se que isso não foi projetado para essa pesquisa, não comprometendo os resultados por hora apresentados. Quanto à investigação empírica, aponta-se como limitação o fato de não reunir evidências de validade externa da EFC, no entanto, justifica-se como evidências psicométricas complementares da escala original e primeiras que consideraram a região Nordeste.

Como indicação de estudos futuros e avanços na área, sugere-se a inclusão de outras escalas em coletas similares, a fim de averiguar a validade externa da medida, conhecer a relação e influência de variáveis sociais, individuais (e.g., valores humanos, personalidade) e demográficas (e.g., sexo, idade) na prevalência das forças de caráter. Ademais, uma coleta realizada de maneira probabilística, contando com uma amostra mais diversificada, oriunda de diferentes regiões do Brasil, contemplaria o avanço acerca do perfil dos pontos fortes do brasileiro. Poderia realizar, também, levantamento de informações com auxílio de uma instrumentalização para além de escalas psicométricas, podendo ser realizadas entrevistas, grupos focais e observações que avaliassem especificamente o uso das forças no cotidiano.

Dessa forma, acredita-se que seria possível obter um panorama robusto acerca das qualidades positivas das pessoas, abarcar informações completas dos benefícios experienciados por quem faz uso de suas forças no dia-a-dia (e.g., sensação de excitação, descobertas, revigoração) e consequentemente ser socialmente reconhecido como uma pessoa com um bom 
caráter, ou seja, que reúne e externaliza pontos positivos e agregadores tanto no nível individual, quanto social (Peterson \& Seligman, 2004; Seijts et al., 2017). Além de planejar intervenções que considere a eficácia das forças na superação de situações difíceis (Proyer, Gander, Wellenzohn, \& Ruch, 2015; Renshaw \& Steeves, 2016) e contribuir com o avanço de estudos que versam sobre construtos positivos (Pires et al., 2015; Reppold et al., 2015;).

\section{Conclusões}

Conclui-se que a presente investigação reforçou as qualidades psicométricas da EFC, disponibilizando uma alternativa parcimoniosa para pesquisadores interessados em utilizá-la no contexto nordestino. Ademais, proporciona discussões acerca do mapeamento do caráter, a partir da prevalência das forças, corroborando com a literatura especializada na área quando identificou influência da cultura e região no ranking das 24 forças. Em síntese, a pesquisa aumentou o arcabouço teórico da temática e refletiu sobre a importância de fazer uso das forças individuais a fim de ter qualidade de vida, vivenciando um funcionamento saudável em todos os âmbitos.

\section{Referências}

Azañedo, C. M., Fernández-Abascal, E. G., \& Barraca, J. (2014). Character strengths in Spain: Validation of the Values in Action Inventory of Strengths (VIA-IS) in a Spanish sample. Clínica y Salud, 25(2014), 123-130. doi: 10.1016/j.clysa.2014.06.002

Boe, O., Bang, H., \& Nilsen, F. A. (2015). Experienced military officer's perception of important character strengths. Procedia - Social and Behavioral Sciences, 190(2015), 339-345. doi: 10.1016/j.sbspro.2015.05.008

Brdar, I., \& Kashdan, T. B. (2010). Character strengths and wellbeing in Croatia: An empirical investigation of structure and correlates. Journal of Research in Personality, 44(1), 151154. doi:10.1016/j.jrp.2009.12.001

Breakwell, G. M., Hammond, S., Fife-Schaw, C., \& Smith, J. A. (Orgs.). (2010). Métodos de pesquisa em psicologia ( $3^{\mathrm{a}}$ ed). Porto Alegre, RS: Artmed.

Brock, R. L., Barry, R. A., Lawrence, E., Dey, J., \& Rolffs, J. (2012). Internet administration of paper-and-pencil questionnaires used in couple research: Assessing psychometric equivalence. Assessment, 19(2), 226-242. doi: 10.1177/1073191110382850

Cohen, R. J., Swerdlik, M. E., \& Sturman, E. D. (2014). Testagem e Avaliação Psicológica: Introdução a Testes e Medidas. ( $8^{\circ}$ ed) São Paulo: AMGH.

Couto R. N., \&. Fonsêca P. N. (2019). Character strengths in the Brazilian northeast region: contributions of personality beyond age and sex. Estudos de Psicologia (Campinas), 36, e18137. doi: 10.1590/1982-0275201936e180137

Damásio, B. F. (2012). Uso da análise fatorial exploratória em psicologia. Avaliação Psicológica, 11(2), 213-228.

Díaz-Loving, R. (2005). Emergence and contributions of a Latin American indigenous social psychology. International Journal of Psychology, 40(4), 213-227. doi: 10.1080/00207590444000168

Freitas, E. R., Barbosa, A. J. G., \& Neufeld, C. B. (2016). Forças do caráter de idosos: Uma revisão sistemática de pesquisas empíricas. Psicologia em pesquisa, 10(2), 85-93. doi: $10.24879 / 201600100020054$ 
Gonçalves, H. F., \& Araújo, J. B. (2015). Evolução histórica e o quadro socioeconômico do Nordeste brasileiro nos anos 2000. Revista do Desenvolvimento Regional - Faccat Taquara/RS, 12(1). 193-204.

Haridas, S., Bhullar, N., \& Dunstan, D. A. (2017). What's in character strengths? Profiling strengths of the heart and mind in a community sample. Personality and Individual Differences, 113(2017), 32-37. doi: 10.1016/j.paid.2017.03.006

Harzer, C., \& Ruch, W. (2013). The application of signature character strengths and positive experiences at work. Journal of Happiness Studies, 14(3), 965-983. doi: 10.1007/s10902012-9364-0

Hofstede, G. (1991). Cultures and Organizations: software of the mind. London: McGraw-Hill.

Hofstede, G., Hofstede, G.J., \& Minkov, M., (2010). Cultures and organizacións: Software of the mind. New York: McGraw Hill

Lorenzo-Seva, U., Timmerman, M. E., \& Kiers, H. A. L. (2011). The Hull Method for Selecting the Number of Common Factors. Multivariate Behavioral Research, 46(2), 340-364. doi: $10.1080 / 00273171.2011 .564527$

Lorenzo-Seva, U. \& Ferrando, P. J. (2013). FACTOR 9.2: A Comprehensive Program for Fitting Exploratory and Semiconfirmatory Factor Analysis and IRT Models. Applied Psychological Measurement, 37(6), 497-498. doi: 10.1177/0146621613487794

McGrath, R. E. (2015): Character strengths in 75 nations: An update. The Journal of Positive Psychology: Dedicated to furthering research and promoting good practice, 10(1), 41-52. doi: 10.1080/17439760.2014.888580

Neto, J., Neto, F., \& Furnham, A. (2014). Gender and Psychological Correlates of Self-rated Strengths Among Youth. Social Indicators Research, 118(1), 315-327. doi: 10.1007/s11205-013-0417-5

Noronha, A. P. P., \& Barbosa, A. J. G. (2016). Forças e virtudes: escala de Forças de Caráter. In C. S. Hutz (Org.), Avaliação em psicologia positiva: técnicas e medidas (pp. 21-43). São Paulo: Hogrefre.

Noronha, A. P. P., \& Batista, H. H. V. (2017). Escala de forças e estilos parentais: estudo correlacional. Estudos Interdisciplinares em Psicologia, Londrina, 8(2)02-19. doi:10.5433/2236-6407.2016v8n2p02

Noronha, A. P. P. \& Batista, H. H. V. (2020a). Relações entre forças de caráter e autorregulação emocional em universitários brasileiros. Revista Colombiana de Psicología, 29(1), 73-86. doi: $10.15446 / . v 29 n 1.72960$

Noronha, A. P. P. \& Batista, H. H. V. (2020b). Análise da estrutura interna da Escala de Forças de Caráter. Ciencias Psicológicas, 14(1), 1-12. doi: 0.22235/cp.v14i1.2150

Noronha, A. P. P., \& Campos R. R. F. (2018). Relationship between character strengths and personality traits. Estudos de Psicologia (Campinas), 35(1), 29-37. doi: 10.1590/198202752018000100004

Noronha, A. P. P., Dellazzana-Zanon, L. L., \& Zanon C. (2015). Internal structure of the Strengths and Virtues Scale in Brazil. Psico USF, 20(2), 229-235. doi: 10.1590/141382712015200204

Noronha, A. P. P. \& Martins, D. F. (2016). Associações entre Forças de Caráter e Satisfação com a Vida: Estudo com Universitários. Acta Colombiana de Psicología, 19(2), 97-103. doi: 10.14718/ACP.2016.19.2.5

Noronha, A. P. P., Silva, E. N., \& Rueda, F. J. M. (2018). Relaciones entre fortalezas del carácter y percepción de apoyo social. Ciencias Psicológicas,12(2), 187-193. doi: 10.22235/cp.v12i2.1681 
Noronha, A. P. P. \& Zanon, C. (2018). Strenghts of Character of Personal Growth: Structure and Relations with the Big Five in the Brazilian Context. Paidéia (Ribeirão Preto), 28(e2822). doi: 10.1590/1982-4327e2822

Oliveira, C., Nunes, M. F. O., Legal, E. J., \& Noronha, A. P. P. (2016) Bem-Estar Subjetivo: estudo de correlação com as Forças de Caráter. Avaliação Psicológica,15(2), 177-185. doi: 0.15689/ap.2016.1502.06

Park, N., Peterson, C., \& Seligman, M. E. P. (2006). Character strengths in fifty-four nations and the fifty US states. The Journal of Positive Psychology, 1(3), 118-129. doi: $10.1080 / 17439760600619567$

Peterson, C., \& Seligman, M. E. P. (2004). Character strengths and virtues: a handbook and Classification. Washington, DC: American Psychological Association.

Petkari, E., \& Ortiz-Tallo, M. (2016). Towards Youth Happiness and Mental Health in the United Arab Emirates: The Path of Character Strengths in a Multicultural Population. Journal of Happiness Studies, 19, 333-350. doi: 10.1007/s10902-016-9820-3

Pires, J. G., Nunes, M. F. O., \& Nunes, C. H. S. (2015). Instrumentos Baseados em Psicologia Positiva no Brasil: uma Revisão Sistemática. Psico-USF, Bragança Paulista, 20(2), 287295. doi: 10.1590/1413-82712015200209

Proyer, R. T., Gander, F., Wellenzohn, S., \& Ruch, W. (2015). Strengths-based positive psychology interventions: A randomized placebo-controlled online trial on long-term eff ects for a signature strengths- vs. a lesser strengthsintervention. Frontiers in Psychology, 9(456), 1-14. doi: 10.3389/fpsyg.2015.00456.

Niemiec, R. M. (2013). VIA Character Strengths: Research and Practice (The First 10 Years). Em H. H. Knoop \& A. Delle-Fave (Eds.), Well-Being and Cultures (pp. 11-29). New York, NY: Springer.

Nunnally, J. C. (1978). Psychometric theory. New York: McGraw-Hill Inc.

Renshaw, T., \& Steeves, R. M. O. (2016). What good is gratitude in youth and schools? A systematic review and meta-analysis of correlates and intervention outcomes. Psychology in the Schools, 53(3), 286-305. doi: 10.1002/pits.21903.

Reppold, C. T., Gurgel, L. G., \& Schiavon, C. C. (2015). Research in Positive Psychology: a Systematic Literature Review. Psico-USF, Bragança Paulista, 20(2), 275-285. doi: 10.1590/1413-82712015200208

Romero, N. A. R. C., Guajardo, J. G., \& Sanchez, A. M. (2016). Las fortalezas de los mexicanos, un análisis desde la autopercepción. Revista Iberoamericana de Psicología: ciencia y tecnología, 9(1), 73-84.

Ruch, W., Martínez-Martí, M. L., Proyer, R. T., \& Harzer, C. (2014). The character strengths rating form (CSRF): development and initial assessment of a 24-Item rating scale to assess character strengths. Personality and Individual Differences, 68, 53-58. doi: 10.1016/j.paid.2014.03.042

Santana, D. L. de; Mendes, G. A.; Mariano, A. M. (2014). Estudo das dimensões culturais de Hofstede: análise comparativa entre Brasil, Estados Unidos e México. C@LEA - Revista Cadernos de Aulas do LEA, Ilhéus, 3, 1-13.

Seligman, M. E. P., \& Csikszentmihalyi, M. (2000). Positive psychology: an introduction. American Psychologist, 55, 5-14. doi: 10.1037/0003-066X.55.1.5

Seligman, M. E. P., Steen, T. A., Park, N., \& Peterson, C. (2005). Positive psychology progress: Empirical validation of interventions. American Psychologist, 60, 410-421. doi: 10.1037/0003-066X.60.5.410 
Seibel, B. L., DeSousa, D., \& Koller, S. H. (2015). Adaptação brasileira e estrutura fatorial da escala 240-item VIA Inventory of Strengths, Psico-USF, 20(3), 371-383. doi: $10.1590 / 1413-82712015200301$

Seijts, G., Crossan, M., \& Carleton, E. (2017). Embedding leader character into HR practices to achieve sustained excellence. Organizational Dynamics, 46 (2017), 30-39. doi: 10.1016/j.orgdyn.2017.02.001

Tabachnick, B. G., \& Fidell, L. S. (2013). Using multivariate statistics (6 ${ }^{\mathrm{a}}$ ed.). Nova Iorque: Allyn \& Bacon

O presente artigo é oriundo do projeto de tese de doutorado do primeiro autor, sob orientação da segunda autora.

Financiamento: O presente artigo teve o apoio da CAPES, que concedeu bolsa de doutorado ao primeiro e terceiro autor e ao CNPq por meio da bolsa de produtividade a segunda autora. Aproveitamos para demonstrar nossa gratidão a essas instituições.

Participação dos autores: a) Planejamento e concepção do trabalho; b) Coleta de dados; c) Análise e interpretação de dados; d) Redação do manuscrito; e) Revisão crítica do manuscrito. R.N.C. contribuiu em a,b,c,d,e; P.N.F. em a,b,c,d,e; P.G.N.S. em c,d,e; P.C.B.M em c,d,e.

Editora científica responsável: Dra. Cecilia Cracco 